\title{
Separation of Ground and Low Vegetation Signatures in LiDAR Measurements of Salt-Marsh Environments
}

\author{
Cheng Wang, Massimo Menenti, Marc-Philippe Stoll, Alessandra Feola, Enrica Belluco, and Marco Marani
}

\begin{abstract}
Light detection and ranging (LiDAR) has been shown to have a great potential in the accurate characterization of forest systems; however, its application to salt-marsh environments is challenging because the characteristic short vegetation does not give rise to detectable differences between first and last LiDAR returns. Furthermore, the lack of precisely identifiable references (e.g., buildings, roads, etc.) in marsh areas makes the registration and bias correction of the LiDAR data much more difficult than in conventional urban- or forested-area applications. In this paper, we introduce reliable methods to remove random and systematic errors and to register raw data, as well as a new procedure, to determine the optimal filter window size to separate ground and canopy returns. A limited amount of field observations is used to determine the size of the filtering window which produces the minimally biased estimates of the digital terrain model (DTM). The digital surface model (DSM, representing the canopy top) is then obtained in a similar manner, and the digital vegetation model (DVM, representing the vegetation height) is computed as the difference between the DSM and the DTM. We apply this procedure to a study marsh within the Venice Lagoon, Italy, and obtain a high-accuracy DTM. The error $\left(z_{-} L i D A R-z_{-}\right.$field $)$is $2.2 \mathrm{~cm}$, with a standard deviation of $6.4 \mathrm{~cm}$. The comparison of the estimated DVM with field observations shows an underestimation of the height of the canopy top $(17.7 \mathrm{~cm}$, on average). The height of the lowest canopy elements (e.g., basal leaves), however, is significantly correlated to the LiDAR-derived DVM, showing that this contains useful information on the canopy structure.
\end{abstract}

Manuscript received November 25, 2008. First published March 27, 2009; current version published June 19, 2009. This work was supported in part by the European Commission through the 5th Framework Program's "Tidal Inlet Dynamics and Environment" (TIDE) Project EVK3 CT 2001-00064, by the PRIN 2006 Modelli dell'evoluzione ecomorfologica di bassifondi e barene lagunari, and by the VECTOR-FISR 2002 project, CLIVEN research line.

C. Wang was with the Laboratoire des Sciences de l'Image, de l'Informatique et de la Teledetection, Université Louis Pasteur, 67412 Illkirch, France, and was also with the State Key Laboratory of Remote Sensing Science, Institute of Remote Sensing Applications of the Chinese Academy of Sciences-Beijing Normal University, Beijing 100101, China. He is now with Boise Center Aerospace Laboratory, Idaho State University, 83702 Boise.

M. Menenti was with the Laboratoire des Sciences de l'Image, de l'Informatique et de la Teledetection, Université Louis Pasteur, 67412 Illkirch, France. He is now with the Department of Earth Observation, Delft University of Technology, 2600 GB Delft, The Netherlands.

M.-P. Stoll is with the Laboratoire des Sciences de l'Image, de l'Informatique et de la Teledetection, Université Louis Pasteur, 67412 Illkirch, France.

A. Feola was with the Dipartimento di Ingegneria Idraulica, Marittima, Ambientale, e Geotecnica, Università di Padova, 35131 Padova, Italy. She is now with the Superior Institute for Environmental Protection and Research, 30100 Venice, Italy.

E. Belluco and M. Marani are with the Dipartimento di Ingegneria Idraulica Marittima Ambientale e Geotecnica, Università di Padova, 35131 Padova, Italy.

Color versions of one or more of the figures in this paper are available online at http://ieeexplore.iee.org.

Digital Object Identifier 10.1109/TGRS.2008.2010490
Index Terms-Digital terrain model (DTM), digital vegetation model (DVM), light detection and ranging (LiDAR), salt-marsh environments.

\section{INTRODUCTION}

$\mathbf{M}$ ARSHES are ubiquitous landforms in estuaries and lagoons and perform important ecosystem functions, influenced jointly by hydrological, morphological, and biological processes [1], [2]. Marshes are characterized by a complex channel network, relatively flat terrain, and are covered by low, dense, and heterogeneous halophytic vegetation [3]. The biologically relevant properties of salt marshes are chiefly determined by salinity, soil saturation, redox potential, and nutrients and are locally linked to soil elevation and characteristics [4]. Indeed, a fundamental problem in intertidal studies is the identification of the relationship between terrain morphology, ground elevation, and the spatial distribution of vegetation species, in order to clarify the complex biogeomorphological processes governing the temporal evolution of tidal environments [5]-[9]. A better understanding of the relation between salt-marsh vegetation and topography can be gained through high-resolution concurrent observations of the spatial distribution of individual species and of ground elevation [10]. Previous studies showed that multispectral and hyperspectral remote sensing can provide accurate vegetation species maps in tidal environments [6], [11]. Airborne light detection and ranging (LiDAR) technologies developed in the last decade provide unprecedented detailed 3-D descriptions of a variety of vegetative systems [12]-[14]. Laser pulses can partly penetrate through vegetation cover, and ideally, particularly in a sparsely vegetated area, some returns come from the ground. The correct separation of ground and vegetation returns hence allows the mapping of the ground topography. However, it is not a simple task to determine which laser returns are ground returns [15], particulary in salt marshes and other low vegetation areas [13]. The effective use of LiDAR measurements in tidal environments requires addressing three main challenges.

1) Marsh areas are covered by dense vegetation, leading to a relatively low chance of laser penetration through the canopy to the ground [13].

2) The relatively few ground returns cannot be easily separated from vegetation returns by sampling the return waveform or by using first and last returns because of the low height of the vegetation (often less than $100 \mathrm{~cm}$ ) compared with the duration and temporal laser separation 
in the LiDAR system. As a consequence, the first and last returns of LiDAR data are practically identical [15].

3) The inaccuracy introduced by the uncertain separation of ground and vegetation returns plus the intrinsic instrument inaccuracies may lead to unacceptably large estimation errors compared with the typically small topographic relief of salt marshes (e.g., on the order of $1 \mathrm{~m}$ in microtidal environments, see [3]).

Based on these challenges, this paper focuses on developing an optimal procedure for producing accurate digital terrain models (DTMs) in salt-marsh areas using airborne LiDAR data and associated field observations. We also explore the potential for establishing digital surface models (DSMs) and digital vegetation models (DVMs).

\section{DESCRIPTION OF LiDAR AND ASSOCIATED DTM/DVM EXTRACTION METHODS}

LiDAR acquires 3-D coordinates of objects on the Earth's surface, allowing for rapid high-resolution measurements of topography over large areas. Commercial airborne LiDAR systems typically measure either one or a small number of surface elevations for a given laser return by identifying, in the return signal, major peaks that represent discrete objects along the path of laser illumination [16]. Modern LiDAR instruments are usually characterized by a very high scanning density and a small footprint, which allow detailed and spatially distributed characterizations of surface elevations.

LiDAR instruments have been effectively used to produce 3-D characterizations of forest ecosystems by exploiting the large separation between the top of the canopy and the ground surface [12], [17]. However, the effectiveness of LiDAR in the presence of dense low vegetation, where differences of a few tens of centimeters make it difficult to discriminate laser returns reflected by the ground and the canopy, has yet to be determined. This evaluation, in turn, requires the assessment of the laser-beam properties that affect laser canopy penetration and thus determine as to what extent vegetation can be effectively discriminated from the ground. These properties include temporal pulsewidth, received pulse broadening due to the detector bandwidth, and the footprint size relative to the canopy gap size. Previous studies [18], [19] indicate that LiDAR technology has the potential of producing DTMs in estuarine marsh areas; however, actual methods of DTM construction were not addressed in these investigations. In addition, an overestimation was generally observed in the LiDAR-derived DTM, likely due to the presence of vegetation and/or its incomplete removal (e.g., [19]).

Many algorithms have been developed for automatic DTM extraction through the separation of ground and nonground laser hits [14], [17], [20]. Most of these algorithms assume ground elevation changes to be relatively gradual and that the ground is locally the lowest reflecting surface. Hence, in many applications, the lowest LiDAR elevation in a local neighborhood is assumed to most likely represent ground elevation [14], [21]. The key step in this filtering method is to determine the size of the filtering window. The selection of the window size impacts the removal of nonground objects and is directly linked to the penetration properties of the laser beam into the canopy, which, in turn, ultimately determines whether vegetation can be reliably discriminated from the ground.

The possibility of deriving vegetation heights (i.e., the DVM) from LiDAR data has mostly been explored in the case of forest ecosystems [22]-[25]. Most of these applications have demonstrated that LiDAR consistently underestimates tree heights. For example, Ronnholm et al. [24] concluded that the highest point on a spruce tree was not measured, even at a sampling density of 50 points $/ \mathrm{m}^{2}$. In a sagebrush steppe environment, another low-height vegetation ecosystem, Streutker and Glenn [14] find that the LiDAR-determined vegetation heights were moderately well correlated to those measured in the field but that the absolute estimation accuracy was relatively low.

The derivation of vegetation height in marsh areas is likely even more difficult because of the typical sparseness of canopy elements and the small extent of their horizontal projection. In this paper, we present a new approach to determine the size of the filtering window by statistical analyses of the field measurements and associated LiDAR data. This method is applied to the LiDAR data in a salt-marsh area to generate a DTM and a DVM.

\section{Materials And Methods}

\section{A. Study Site}

The lagoon of Venice, Italy [see Fig. 1(a)], covers an area of approximately $550 \mathrm{~km}^{2}$ with a mean water depth of about $1.1 \mathrm{~m}$ and a tidal range of $\sim 1.6 \mathrm{~m}$. The lagoon is connected to the Adriatic Sea through three inlets and receives a very modest freshwater input from a few tributaries. This paper analyzes LiDAR data acquired over the San Felice marsh [see Fig. 1(b)], where soil elevation ranges from about $0.01 \mathrm{~m}$ above mean sea level (a.m.s.l.) to $0.68 \mathrm{~m}$ a.m.s.l. (with an average of $0.26 \mathrm{~m}$ a.m.s.l.) [6]. Land cover at San Felice consists of bare soil and four dominant vegetation species: Spartina maritima (Spar.), Sarcocornia fruticosa (Sarc.), Limonium narbonense (Limo.), and Juncus maritimus (Junc.). It is important to briefly describe here the typical structure, height, and density of vegetation species within the study site as these have consequences on the likelihood of ground and vegetation LiDAR returns occurrence. Typical vegetation heights range from 30 to $70 \mathrm{~cm}$ [4]. Spartina maritima has an almost vertical development and typically occurs in moderately dense patches. Soil is quite clearly visible when looking at the vegetation from above (see Fig. 2 for a typical situation in which $40 \%$ of the area is occupied by soil). Limonium narbonense is constituted by basal leaves and taller xylems with flowers, such that its parts with appreciable horizontal projections are located at two quite well-defined and distinct heights. Soil is visible from above but takes up a relatively small portion of the area, e.g., $30 \%$ in the case of Fig. 2, quite representative of typical conditions in the summer. Sarcocornia fruticosa is typically shorter than the remaining species and has a more compact structure. Plants are usually quite densely spaced. As a result, a small portion of soil is usually visible from above during the summer $(20 \%$ in Fig. 2), which, however, increases significantly in winter. Juncus maritimus occurs in clustered bushes such that soil is 


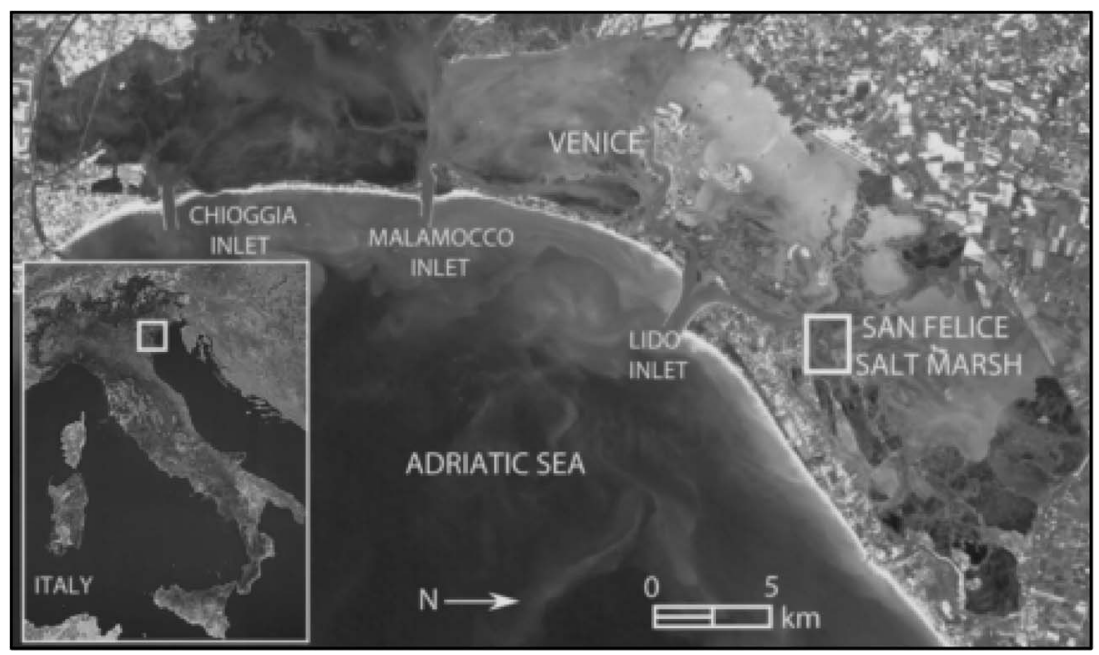

(a)

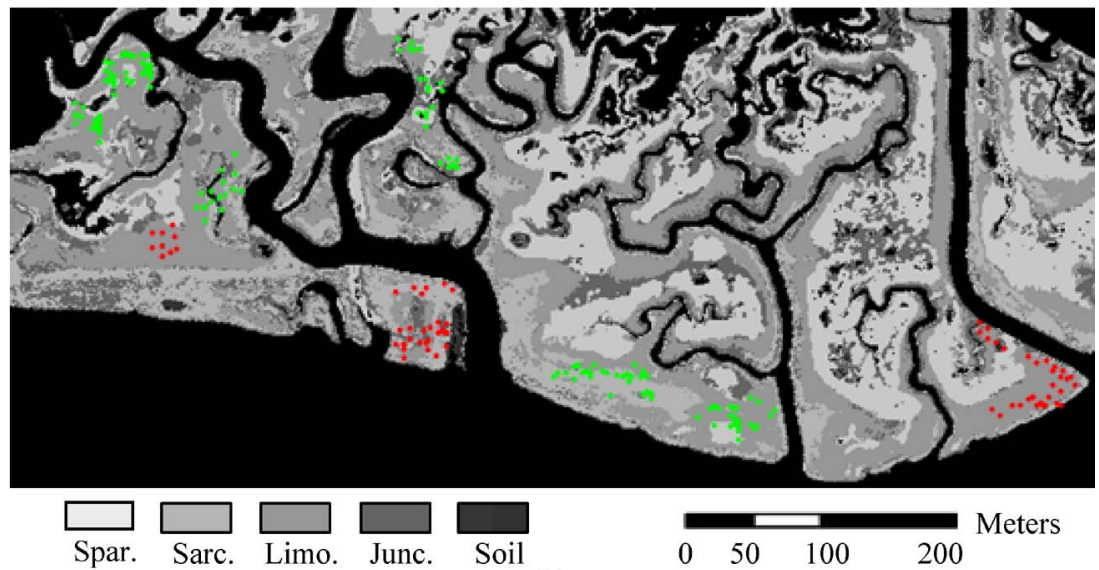

(b)

Fig. 1. (a) Location of San Felice salt marsh, Italy. (b) Classified vegetation map of San Felice study area outlined in (a), with field measurements in green and red.

often visible from above in between the bushes $(30 \%$ of soil in Fig. 2), whereas laser returns are certainly reflected by the canopy within a bush. Considering the thin stems composing a bush, it is not easy to identify a level at which reflection is likely to occur. Notice that, although all the species are perennial, significantly higher fractions of soil are visible in the winter (when the LiDAR acquisition was performed) because of the reduced standing biomass.

\section{B. Differential GPS Reference Data}

Ground reference observations were collected using twoband GPS receivers and subsequent differential corrections [differential GPS (DGPS), with a minimum accuracy of $\pm 1 \mathrm{~cm}$ in all directions], for LiDAR data preprocessing, analysis, and accuracy assessment. All elevations were defined with respect to the local mean sea level and with reference to UTM-WGS84 planar coordinates. Five different DGPS data sets have been used in this paper, namely: 1) DGPS data at a known ground station to differentially correct onboard GPS plane positioning (not explicitly described/used here); 2) 20 DGPS elevation observations of paved surfaces located about $1 \mathrm{~km}$ south of the study marsh. Comparisons of such reference data with LiDAR estimates show no bias and a root-mean-square error (rmse) of $6.7 \mathrm{~cm}$, thus showing that there is no datum offset between LiDAR elevations and DGPS observations used for calibration/validation procedures; 3) DGPS data of soil elevation along two channels/creeks ("north creek" and "south creek" in the following) for the $(x, y)$ planar adjustment of LiDAR data. This data set, acquired in February-March 2002, is composed of 664 point measurements defining the geometry of the "south creek" and 471 point measurements for "north creek"; 4) DGPS data of soil elevation distributed across the study area to define the optimal size of the filtering window for DTM construction. This data set, the calibration data set, is composed of 240 DGPS observations collected during a fourday field campaign in the summer of 2002 (May 22, and 29 and July 15 and 16, 2002) [green points in Fig. 1(b)]; and 5) DGPS ground elevation observations [red points in Fig. 1(b)] used in the quality assessments of LiDAR-derived DTM and DVM. This validation data set is composed of 162 DGPS observations acquired on February 10, 2003, simultaneous with the LiDAR survey. Minimum and maximum canopy heights within a radius $r \approx 20 \mathrm{~cm}$ around each DGPS observation 


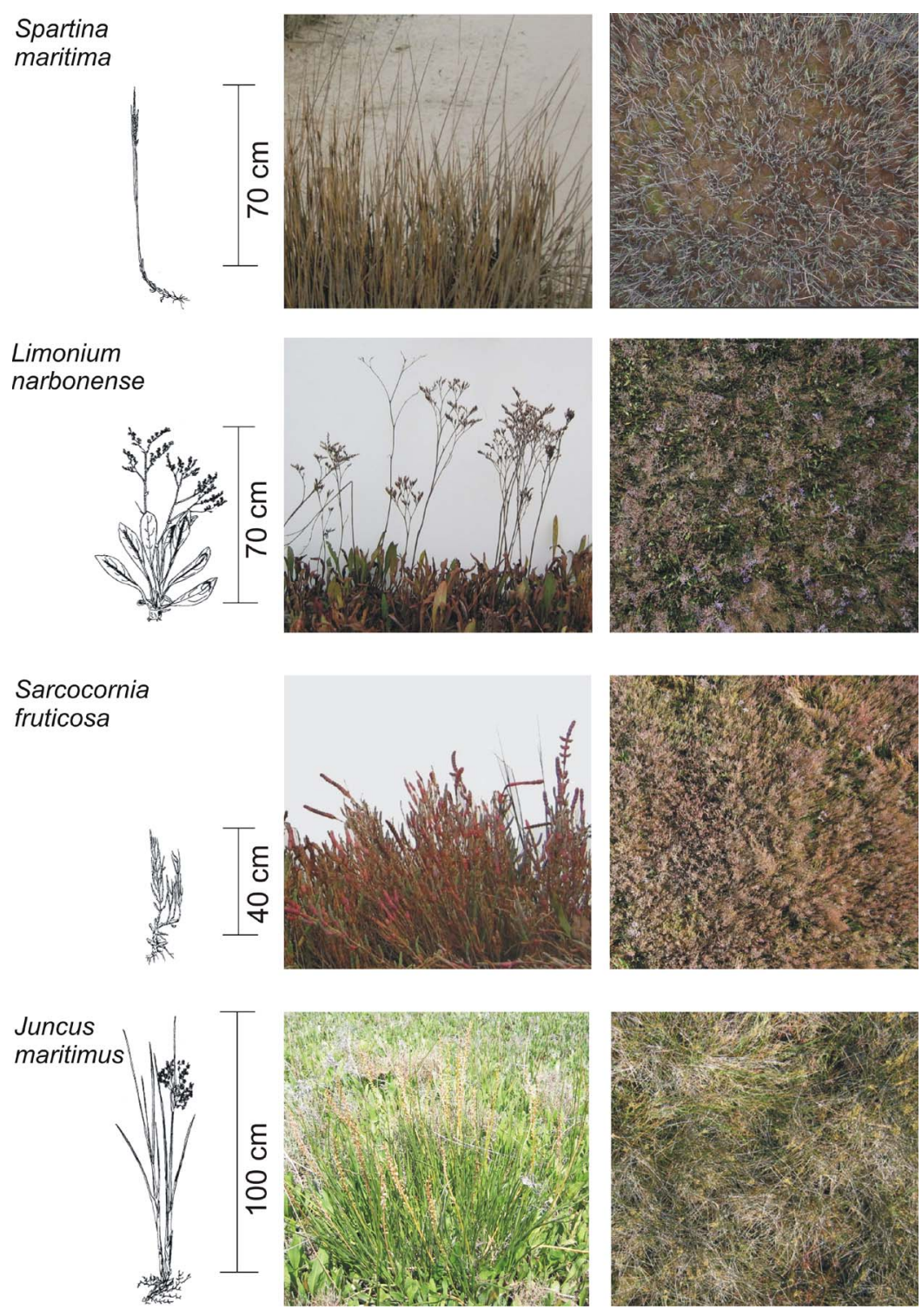

Fig. 2. Typical structure, profile, and density of the main four vegetation species occurring within the study marsh. The first column illustrates the typical canopy structures. The second column presents typical lateral profiles of the canopy, while the last column shows pictures taken with a nadir-looking camera suspended on a pole about $2.5 \mathrm{~m}$ above the ground to illustrate typical densities.

TABLE I

StatisticAl Descriptions of THE Six FLight Lines

\begin{tabular}{ccccccc}
\hline Flight line & 1 & 2 & 3 & 4 & 5 & 6 \\
\hline Area $\left(\mathrm{m}^{2}\right)$ & 106444 & 102735 & 115979 & 115633 & 76818 & 115214 \\
\# of points & 868145 & 797150 & 863248 & 885464 & 616306 & 897108 \\
Density (points $/ \mathrm{m}^{2}$ ) & 8.16 & 7.76 & 7.44 & 7.66 & 8.02 & 7.79 \\
\hline
\end{tabular}

(roughly the area of a LiDAR footprint) were also concurrently measured.

\section{LiDAR Data}

The LiDAR survey was carried out over the San Felice marsh on February 9, 2003, when the relatively low vegetation density (compared with summer months) maximizes the chances that laser pulses are reflected by the ground. The FALCON II sensor, a small-footprint pushbroom laser altimeter developed by the TopoSys Company, Germany, was used and provided both first and last returns at a wavelength of $1560 \mathrm{~nm}$. The system scans across-track lines with a field of view (FOV) of $21.6^{\circ}$, measuring 682 points per line, which gives an instantaneous FOV (IFOV) of $0.5 \mathrm{mrad}$ and an acrosstrack spacing of data points of $0.32 \mathrm{~m}$. The scan rate is up to 330 lines/s. The aircraft speed was $60 \mathrm{~ms}^{-1}$ at a flying height of $450 \mathrm{~m}$ above ground for the acquisitions used in this paper, i.e., with a minimum along-track spacing of data points of $0.18 \mathrm{~m}$ and a footprint size of $22.5 \mathrm{~cm}$. A density of approximately 8 points $/ \mathrm{m}^{2}$ was computed from the acquired LiDAR data (Table I). 
In the case of laser returns reflected by a locally smooth area, the received laser intensity typically quickly rises to a plateau level, remains at that level for some time, and then rapidly decreases to zero. Usually, for all time-recording LiDAR devices (such as the FALCON II system), the time of echo detection does not correspond to the very beginning of the intensity increase; rather, a time lag occurs until the magnitude of the intensity exceeds a threshold value. The FALCON II system uses a more sophisticated method which applies a dynamic threshold. The threshold is calculated from the amplitude of the echo itself and ensures that a return is recorded corresponding to the middle of the interval over which the intensity rises to the maximum value. Returns from a vegetated area are composed of many weak echoes, related to the different reflecting elements illuminated by the beam, with the consequence that the resulting echo will be rather long and the trigger signal will trail the actual leading edge of the first return by a significant time lag. As a consequence, the estimated elevation of the vegetation canopy is likely to be significantly lower than the maximum elevation of the canopy (elevation of the highest detectable plant element). Given the vertical structure of salt-marsh vegetation species (Fig. 2), this makes detection of the upper part of the plants rather unlikely.

A further problem with low intertidal vegetation is related to the fact that the time between the first and the last returns, even when the topmost canopy elements actually give rise to a reflection, is too short to be detected. As a result, the first and the last returns are identical and cannot be used to directly determine the elevation of the canopy and the ground, respectively. In the following, we thus process the last return data (six flight lines covering the whole San Felice area, see Table I) in order to provide a discrimination of vegetation and ground returns.

\section{Preprocessing of LiDAR Data}

Although LiDAR accuracy can be calculated, and it is usually provided by commercial vendors, peculiar characteristics of the site under study and different data collection strategies can influence the nominal accuracy. Thus, empirical studies that take into account these characteristics are always necessary [13], [26]. In this paper, before DTM (or DSM, DVM) extraction, an important preprocessing step is to assess the quality of the raw LiDAR data with the aim of removing or reducing random and systematic errors.

\section{A. Removal of Random Errors and of Unnecessary Information}

Random errors may be present in the data, e.g., due to an occasional malfunctioning of the system, which must first be removed. Furthermore, returns from water bodies, which must first be removed. Furthermore, returns from channeled areas exposed by the low tide must also be removed as this study focuses on the observation of the marsh platform and of its vegetation. Field observations in San Felice indicate that the surface elevation of almost the entire area is less than $1 \mathrm{~m}$

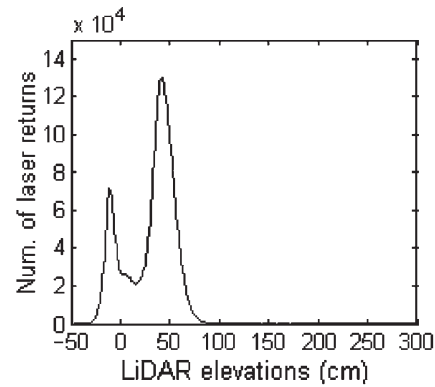

(a)

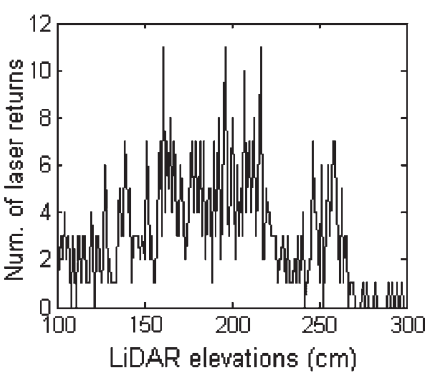

(b)
Fig. 3. Frequency distribution of the LiDAR elevations in the range of (a) $-50-300 \mathrm{~cm}$ a.m.s.l. and (b) $100-300 \mathrm{~cm}$ a.m.s.l.

a.m.s.l., with the exception of a few navigation poles with elevations up to $3 \mathrm{~m}$ in the main channels. The frequency distribution of LiDAR elevations for the whole study site [Fig. 3(a)] clearly shows the presence of two distinct peaks; one relates to marsh areas with an elevation in the $15-90-\mathrm{cm}$ a.m.s.l. range, and the other is due to water (or channel bottom) returns below the mean sea level (the tidal level was about $-15 \mathrm{~cm}$ a.m.s.l. at the time of data acquisition). An additional minor peak [Fig. 3(b)] is also present in the $1-3-\mathrm{m}$ range, which is likely caused by navigation poles in the channel or by other small objects (e.g., huts or rare taller shrubs) on the marsh surface. A detailed analysis of the raw data shows that only 66 LiDAR elevations lie between 3 and $450 \mathrm{~m}$ (flying altitude). These records are likely due to random errors in the LiDAR system or to objects on the surface (e.g., they could possibly come from birds or other features in the airshed) and should be removed from the data set.

If the laser beam is reflected by a still water surface, except for the nadir or near-nadir scan position, a weak or no return will be detected by the near-infrared LiDAR system because of specular reflection. However, wind-generated waves and ripples are often present on the water surface, and thus, the reflected laser beam has a nonzero probability of returning toward the sensor (Fig. 3(a), also see [27]). Since the aim of this paper is to establish DTM/DVM for the salt-marsh area, laser returns from water bodies are undesired information and have been removed by eliminating all laser returns with an elevation $z<0 \mathrm{~m}$ a.m.s.l.

\section{B. Estimation of Planimetric Offsets ( $x-y$ Coordinates)}

The absolute coregistration of LiDAR with GPS observations is difficult because of uncertainties in determining the laser pointing angle and the airplane position at any given time. Furthermore, no easily identifiable objects are present in marsh areas, such as buildings and roads, so that an alternative method to improve the relative positioning of the flight lines and overall absolute geocoding of the elevation data must be devised. To do so, we look for the planar correction that maximizes the correspondence between GPS observations (considered to be exact in all three coordinates, due to their small uncertainty) and raw LiDAR data. First, each GPS observation $\left(x_{i}^{\mathrm{G}}, y_{i}^{\mathrm{G}}, z_{i}^{\mathrm{G}}\right)$ was associated to the nearest LiDAR point $\left(x_{i}^{\mathrm{L}}, y_{i}^{\mathrm{L}}, z_{i}^{\mathrm{L}}\right)$, and 

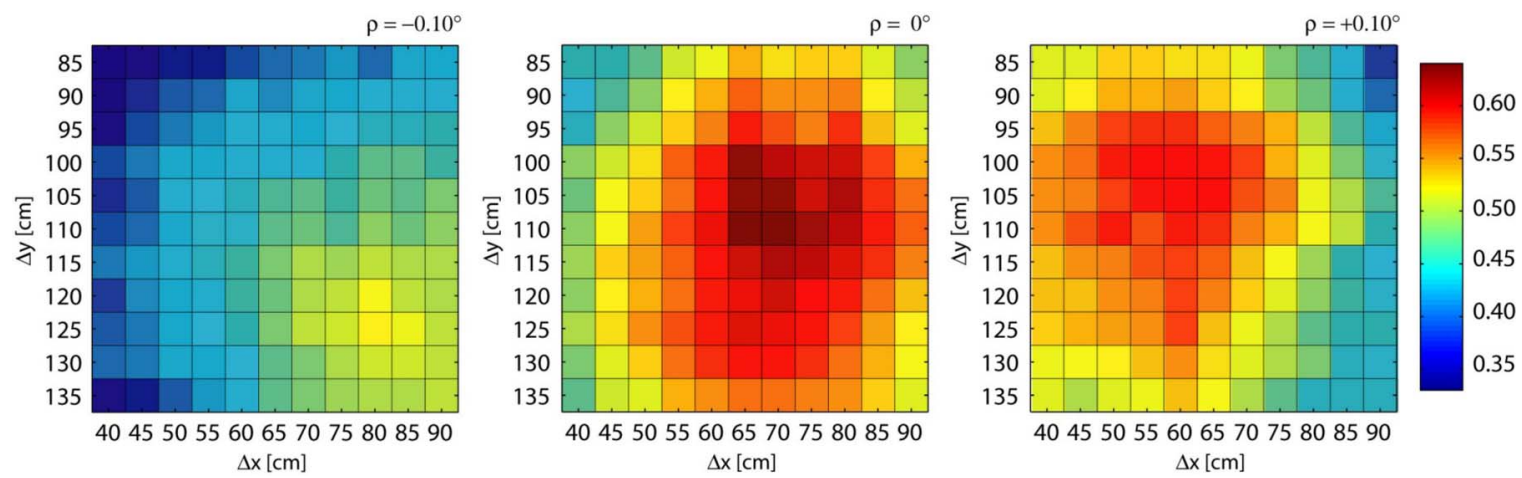

Fig. 4. Correlation coefficient as a function of a generic planar displacement $(\Delta x, \Delta y, \rho)$ for a sample LiDAR flight line, composed of a translation in the $x, y$ plane and of a rigid rotation $\rho$.
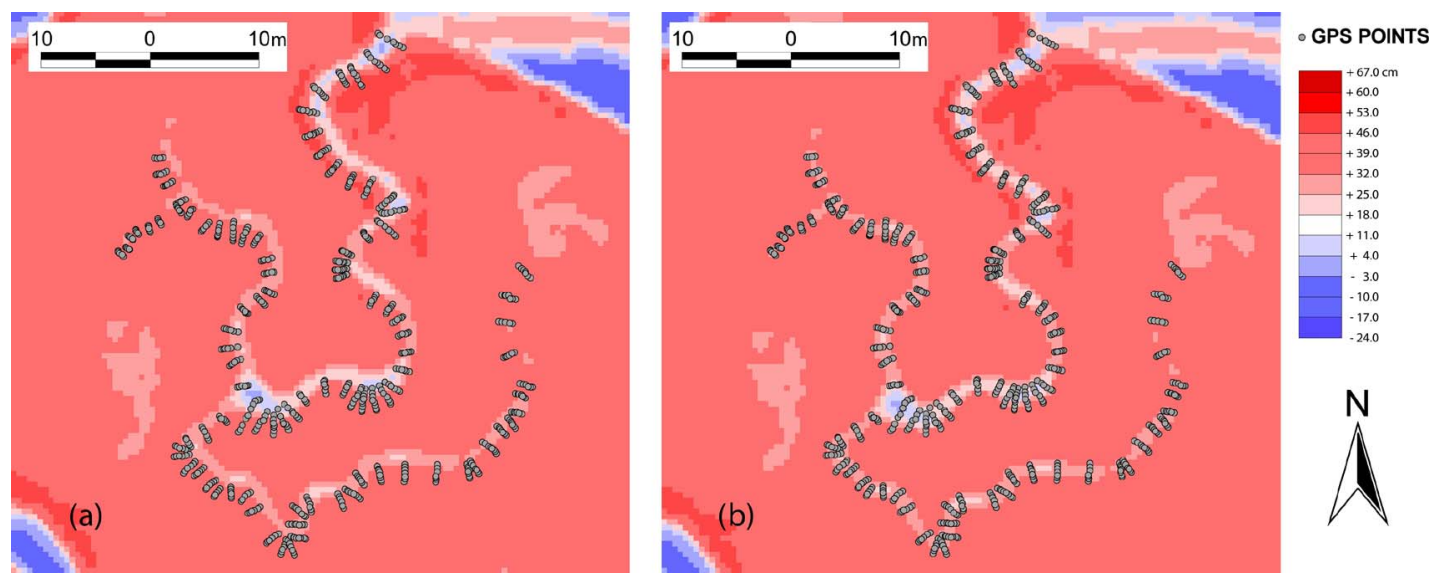

Fig. 5. Planar correspondence between LiDAR and DGPS observations for the "south creek." The dots in the two panels show the position of the DGPS observations of creek cross sections. (a) Before the correction, there is a clear difference in the position of the creek as given by (color-coded elevation, white showing the low-elevation creek bottom) LiDAR data and (DGPS cross sections marked by sequences of solid circles) its "true" position. (b) Match between the two data sets is quite satisfactory after a translational and rotational adjustment maximizing the correlation between the two data sets.

then, the correlation among all such pairs of LiDAR and GPS observations was computed as

$$
C=\frac{\sum_{i}^{N}\left(z_{i}^{\mathrm{G}}-\overline{z^{\mathrm{G}}}\right)\left(z_{i}^{L}-\overline{z^{\mathrm{L}}}\right)}{\sqrt{\sum_{i}^{N}\left(z_{i}^{\mathrm{G}}-\overline{z^{\mathrm{G}}}\right)^{2}} \cdot \sqrt{\sum_{i}^{N}\left(z_{i}^{\mathrm{L}}-\overline{z^{\mathrm{L}}}\right)^{2}}}
$$

where $C$ is the correlation coefficient and $N$ is the number of LiDAR observations within the flight line considered.

If a planar correction composed of a translation $(\Delta x, \Delta y)$ and a rigid rotation $\rho$ (Fig. 4) is applied to the LiDAR data, the correlation coefficient becomes a function $C(\Delta x, \Delta y, \rho)$ which must be maximized in order to yield the best correspondence between the GPS and LiDAR data. This procedure was applied independently to each flight line; however, the values of the corrections resulting from the correlation maximization were all very close. The optimal translation corrections were within $10 \mathrm{~cm}$, and the rotation corrections were within fractions of a degree. A single correction with $\Delta x=65 \mathrm{~cm}, \Delta y=110 \mathrm{~cm}$, and $\rho=0^{\circ}$ was thus adopted for all flight lines. These correction parameters corresponded to a well-located maximum of the correlation (Fig. 4). The correction yielded a significant improvement in the registration of the data, as shown by com- paring the position of topographic features, such as tidal creeks, as mapped by GPS and LiDAR data (Fig. 5).

\section{Estimation of Elevation Offsets}

An overlap analysis method [28] was proposed to estimate the elevation discrepancies of laser returns coming from different flight paths. The overlap analysis, which we use here to estimate the height offset of raw LiDAR data, is based on the comparison of a large number of LiDAR measurements in portions of the area covered by two consecutive and overlapping flight lines. Following this approach, more than 3000 square tiles (each covers a $3 \mathrm{~m} \times 3 \mathrm{~m}$ area) were considered within the overlapping flight lines areas. The tile size was decided on the basis of experiments in which the area was varied from 1 to $100 \mathrm{~m}^{2}$. For each tile within an overlapping area, the mean elevation and its standard deviation were calculated. These values were then averaged over all tiles within each flight line to yield estimates of mean elevation and standard deviation for all flight strips. For any pair of overlapping strips, the difference between such overall mean elevation values (one for each strip) was taken to be the relative height offset [28]. Considering all possible pairs of flight strips, a least squares technique was used to obtain the set of flight-strip offsets which minimizes the mean-square residual offset. 
TABLE II

SumMary of ERror CORRECTIONS PERFORMED ON THE RAW LiDAR DATA

\begin{tabular}{cccc}
\hline \multirow{2}{*}{ Flight lines } & Edited data & \multicolumn{2}{c}{ Systematic errors $(\mathrm{cm})$} \\
\cline { 3 - 4 } & & $\mathrm{x}$-y offset & $\mathrm{z}$ offset \\
\hline 1 & & 1 \\
2 & Number of laser returns & $\Delta x=-65$ & 1 \\
3 & reduced from 28711 to & -1 \\
4 & 21428. & $\Delta y=-110$ & -1 \\
5 & $(\sim 25 \%$ reduction) & & -2 \\
6 & & & 2 \\
\hline
\end{tabular}

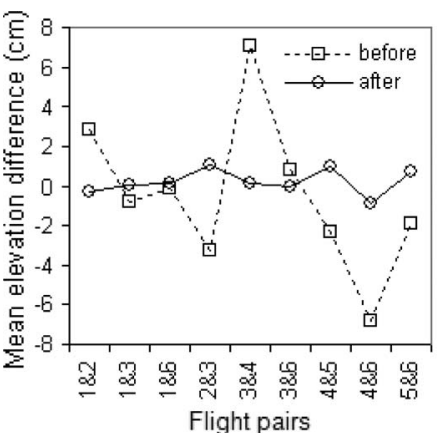

(a)

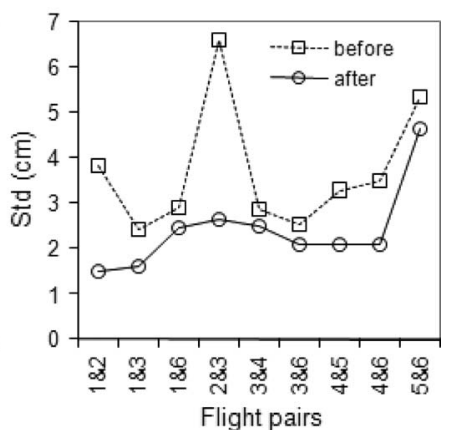

(b)
Fig. 6. Comparison of pre- and postadjustment data sets for (a) mean elevation difference and (b) standard deviation.

In the present case, the six flight lines produced nine overlaps of varying areas. Statistical results from these overlaps indicate the following: 1) $50.19 \%$ of the whole area was covered by at least two flight lines; 2) no laser returns are presented in 14.29\% of the whole area; and 3) most no-return areas in the study site are covered by water bodies (channels and creeks).

\section{Evaluation of Data Correction}

Quality evaluation of the raw LiDAR data was performed on all six flight lines, and Table II summarizes random and systematic errors emerging from the analysis. Approximately $25 \%$ of the laser returns, including both erroneous returns (water and flying-object reflected returns) were removed from the raw LiDAR data. The last two columns in Table II indicate the planimetric corrections determined by (1) and elevation offset which resulted from the overall adjustment procedure.

After data editing and removal of systematic errors, a new LiDAR data set was produced by combining these six flight lines and taking into account their respective elevation offset (Table II). The data quality is significantly improved (Fig. 6). The range of mean differences of the nine overlapping pairs is reduced from about \pm 8 to $\pm 1 \mathrm{~cm}$. Similarly, the standard deviations are minimized.

\section{DTM AND ITS EVALUATION}

\section{A. Ground Echo Identification}

Based on the improved LiDAR data set and field measurements, a new method was introduced for ground return identification. The 240 DGPS points in the calibration data set were used to match the ground-reflected LiDAR elevations, safely assuming that no change in the marsh elevation occurred between field surveys and LiDAR data acquisition. Due to the presence of vegetation, some laser returns have been reflected by the canopy rather than by the ground. The filtering technique, which selects the lowest elevation within a local window, was used to identify ground returns from LiDAR data. The crucial step is to minimize the size of the moving window to ensure a high spatial resolution while still making it large enough to contain a "true" ground hit with a high probability. In order to obtain such an optimal size, we compared the elevation of each DGPS observation to the elevation of surrounding LiDAR points falling within search windows of increasing radius $r$ (here, $r=0.5,1.5,2.5,3.5, \ldots, 6.5 \mathrm{~m}$ ), centered at the DGPS point. The number of LiDAR returns within a circle was approximately $n=\rho \cdot\left(\pi \cdot r^{2}\right)$, where $\rho$ was the echo density of about 7.8 echoes $/ \mathrm{m}^{2}$ (Table I). For each field point and value of $r$, the laser return with the smallest $z_{-} L i D A R$ value was identified and compared with the "actual" elevation $z_{-}$field. When all DGPS observations were considered, a scatter plot of $z_{-}$field versus $z_{-} L i D A R$ was produced for each radius. For the smallest circle $(r=0.5 \mathrm{~m})$, almost all returns came from the canopy, and LiDAR estimates tended to be greater than DGPS observations [Fig. 7(a)]. As the radius of the circle increased, the probability of the ground LiDAR return also increased, and the "optimal" radius was assumed to be the smallest radius giving rise to the best attainable match between $z_{-}$field and $z_{-} L i D A R$. The best match was determined by the overall rmse computed on the basis of all DGPS values. Application of this method on the LiDAR data from the San Felice marsh yielded a radius of $3.5 \mathrm{~m}$, corresponding to a mean error of $2.3 \mathrm{~cm}$ and a standard deviation of $6.5 \mathrm{~cm}$ [Fig. 7(b)]. Such errors are acceptable even in small-relief marshes.

Fig. 8 shows the dependence of the mean and standard deviation of the estimation error on the radius chosen to produce the best LiDAR estimate of ground elevation. The mean error initially rapidly approaches zero but appears to remain roughly constant when $r>3.5 \mathrm{~m}$. Similarly, the error of the standard deviation decreases with increasing radius and reaches an approximately constant value at $r=3.5 \mathrm{~m}$. These results indicated that there indeed exists an optimal size for the search neighborhood in which the lowest LiDAR return represents a true ground reflection.

The aforementioned analysis was applied to all 240 field points in San Felice, and the results show that for about $81 \%$ of these field points, i.e., 194 out of the total 240 points, a ground LiDAR return is actually found within $r \leq 3.5 \mathrm{~m}$. We thus adopt $r=3.5 \mathrm{~m}$ as the optimal window size likely to yield at least one ground hit. With the moving step of $1 \mathrm{~m}$, Fig. 9(a) (also with a 1-m resolution) shows the whole ground returns in the study site. Out of a total of 148793 nonwater pixels, 26633 LiDAR returns are identified as ground returns in the study area, i.e., $18 \%(=26633 / 148793)$ pixels have the ground returns. Considering the laser scanning density of eight points per pixel (or square meter), only $2.24 \%$ of the laser returns are identified as the ground returns. 


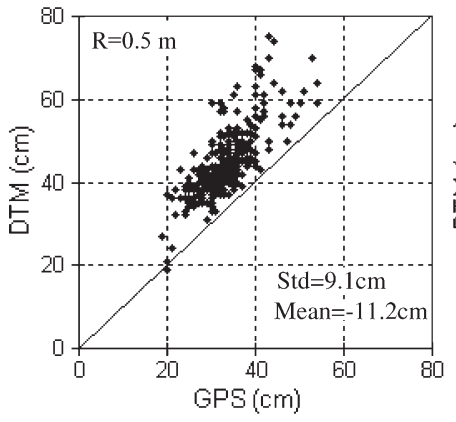

(a)

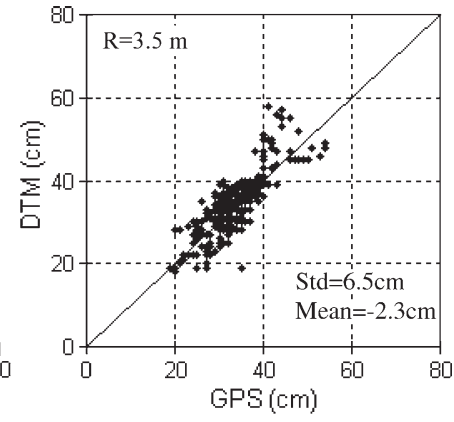

(b)

Fig. 7. Scatter plot of GPS field measurements versus LiDAR estimates of ground elevation using search radius (a) $r=0.5 \mathrm{~m}$ and (b) $r=3.5 \mathrm{~m}$.

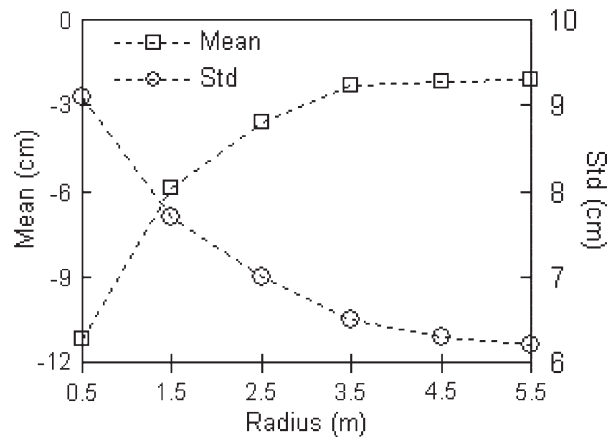

Fig. 8. Mean and standard deviation of the LiIDAR estimation error as functions of the size of the search radius.
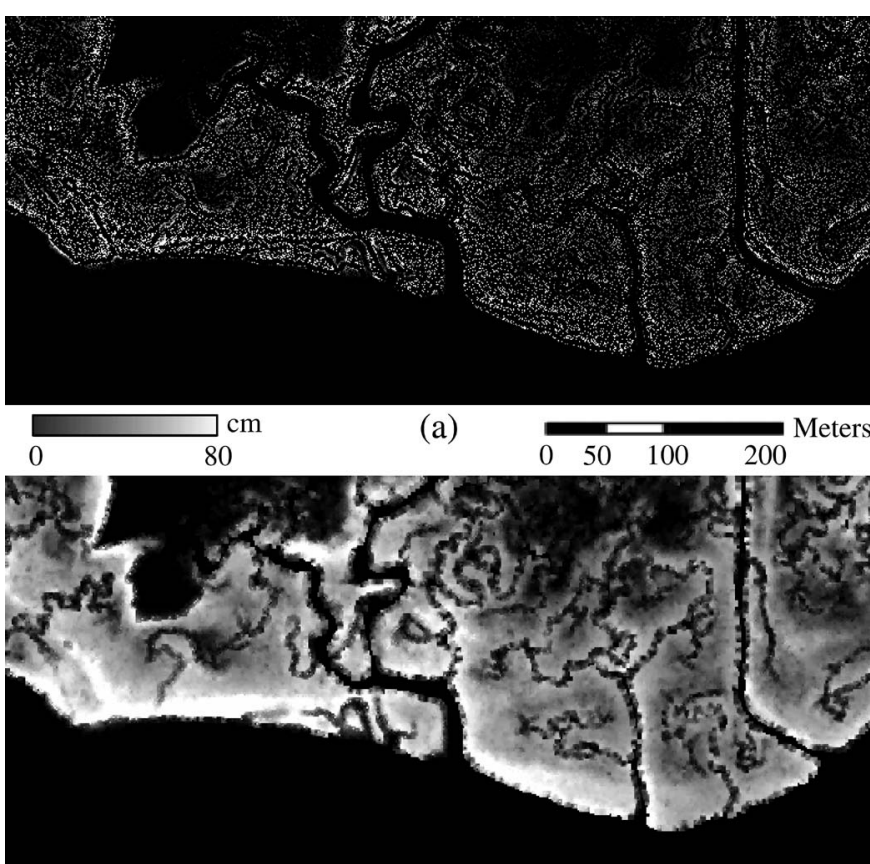

(b)

Fig. 9. (a) Distribution of the ground-reflected LiDAR returns. (b) DTM of the study site by interpolation of the ground returns.

\section{B. Interpolation of Ground Returns}

A raster DTM can now be constructed by resampling the ground-reflected laser returns identified by the filtering technique. Here, an inverse distance-weighting algorithm was used
[29]. A moving 5 pixel $\times 5$ pixel window is adopted in this paper. All identified ground returns [Fig. 9(a)] in the moving local window contribute to the interpolated value, based on their distance from the point where the interpolation is being performed. Fig. 9(b) shows the interpolated DTM with a 1-m spatial resolution.

\section{DTM Validation}

The 162 field DGPS observations in the validation data set (independent from those used to calibrate the algorithm for DTM construction) were used for the accuracy assessment of the extracted DTM. The soil elevations of these field points were compared with the corresponding pixels in Fig. 9(b); a mean difference of $-2.2 \mathrm{~cm}$, with a standard deviation of $6.4 \mathrm{~cm}$, was determined with respect to the 162 validation values.

\section{DSM/DVM CONSTRUCTION AND EVALUATION}

The DSM was constructed using the same techniques used to create the DTM. The LiDAR return corresponding to the maximum elevation value within a search neighborhood (with $r=3.5 \mathrm{~m}$ ) was considered to be the best estimate of the canopy surface elevation. The DVM was then created by subtracting the DTM from the DSM.

As mentioned in Section III-B, for each field point, the maximum and minimum vegetation heights were measured within a circle $r<0.2 \mathrm{~m}$ and were compared with the LiDARderived DVM. The comparison results (Fig. 10) show that an average underestimation of $17.7 \mathrm{~cm}$ occurs with respect to the maximum field-measured vegetation height, while the minimum vegetation height observed in the field is, on average, overestimated by $1.5 \mathrm{~cm}$.

\section{DisCUSSION AND CONCLUSION}

We presented a complete framework for LiDAR data processing and analysis in salt-marsh areas, including error evaluation and correction, data filtering, and DTM/DSM/DVM construction. The main conclusions that result from the application of the proposed methods may be summarized as follows.

1) Vegetation and ground LiDAR returns can be discriminated even when the vegetation height is so short that 


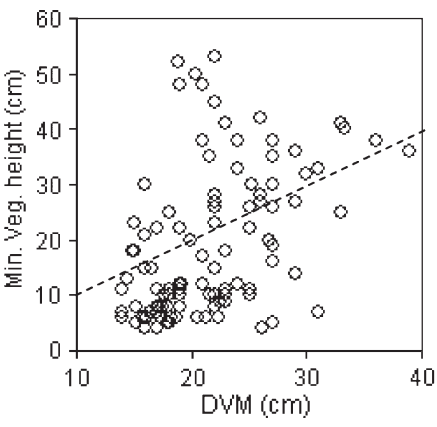

(a)

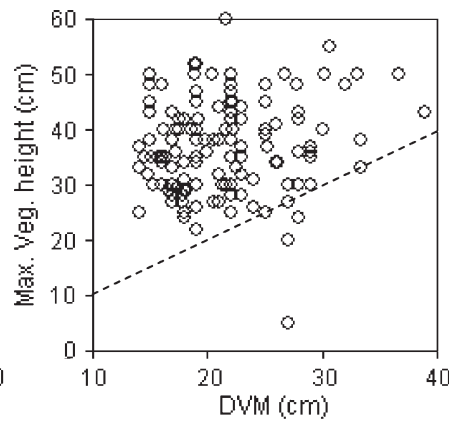

(b)

Fig. 10. Comparisons of LiDAR-determined vegetation heights (or DVM) versus (a) field-measured minimum vegetation heights and (b) field-measured maximum vegetation heights. The dashed line represents a 1:1 correspondence.

differences in the first and the last returns are not detectable. The filtering method used to overcome this problem can be optimized with the aid of a limited number of field reference observations in order to determine the highest spatial resolution (filtering window size), allowing the minimum bias in ground elevation estimates.

2) The DTM obtained in the application exhibits a minimal bias $(-2.2 \mathrm{~cm})$ and a modest rmse of $6.4 \mathrm{~cm}$, comparable with the rmse computed from validations on paved surfaces $(6.7 \mathrm{~cm}$, as described earlier). This implies that airborne LiDAR data can be used to successfully characterize salt-marsh topography even when relief is of the same order of magnitude of that of the nominal instrument error.

3) Considering the sparseness of the upper canopy elements and the lags in the return detection intrinsic to LiDAR systems, the elevation of the canopy top is typically underestimated by the LiDAR systems (Fig. 10). However, it is interesting to note that the average underestimation of the canopy top [17.7 cm, see Fig. 10(b)] is smaller than the typical biases encountered in the LiDAR estimation of forest tree heights (errors ranging from 0.2 to $1.8 \mathrm{~m}$, e.g., [22]-[25]). Considering the short height of marsh vegetation, relative errors of the DVM are, however, much higher in the present case.

4) Although it is difficult to accurately estimate vegetation heights, other vegetation information may be derived from LiDAR in marsh areas. The frequency distribution of LiDAR elevations within a local window provides a statistical description of the canopy structure, although the information from the very top of the canopy is typically missing. We believe that the small underestimation of the minimum canopy height is quite encouraging. Further work (involving the collection of above-ground biomass) is, however, required to determine a quantitative link between LiDAR-derived statistics and biomass. The frequency distributions of LiDAR elevations were approximately normal, as dictated by the random position of the reflecting elements along the vertical direction in the vegetation canopy. Further work should be pursued to link the characteristics of such frequency distributions with the vertical structure of the canopy.

\section{ACKNOWLEDGMENT}

The authors would like to thank Dr. N. Glenn of Idaho State University and three anonymous reviewers for their helpful comments in improving earlier versions of this paper.

\section{REFERENCES}

[1] D. A. Kreeger and M. P. Weinstein, "Special issue on salt marshes: Concepts and controversies," Wetlands Ecol. Manag., vol. 8, no. 2/3, pp. 7778, Jun. 2000.

[2] M. Marani, S. Silvestri, E. Belluco, N. Ursino, A. Comerlati, and O. Tosatto, "Spatial organization and ecohydrological interactions in oxygen-limited vegetation ecosystems," Water Resour. Res., vol. 42, p. W06 D06, May 2006. DOI: 10.1029/2005WR004582.

[3] M. Marani, S. Lanzoni, S. Silvestri, and R. Rinaldo, "Tidal landforms, patterns of halophytic vegetation and the fate of the lagoon of Venice," $J$. Mar. Syst., vol. 51, no. 1-4, pp. 191-210, Nov. 2004.

[4] S. Silvestri, A. Defina, and M. Marani, "Tidal regime, salinity and salt marsh plant zonation," Estuar. Coast. Shelf Sci., vol. 62, no. 1/2, pp. 119130, Jan. 2005.

[5] S. Silvestri, M. Marani, J. Settle, F. Benvenuto, and A. Marani, "Salt marsh vegetation radiometry-Data analysis and scaling," Remote Sens. Environ., vol. 80, no. 3, pp. 473-482, Jun. 2002.

[6] E. Belluco, M. Camuffo, S. Ferrari, L. Modenese, S. Silvestri, A. Marani, and M. Marani, "Mapping salt-marsh vegetation by multispectral and hyperspectral remote sensing," Remote Sens. Environ., vol. 105, no. 1, pp. 54-67, Nov. 2006.

[7] A. D'Alpaos, S. Lanzoni, M. Marani, S. Fagherazzi, and A. Rinaldo, "Tidal network ontogeny: Channel initiation and early development," J. Geophys. Res., vol. 110, no. F2, p. F02001, Apr. 2005. DOI: 10.1029/2004JF000182.

[8] A. D’Alpaos, S. Lanzoni, M. Marani, and A. Rinaldo, "Landscape evolution in tidal embayments: Modeling the interplay of erosion, sedimentation, and vegetation dynamics," J. Geophys. Res., vol. 112, p. F01 008, 2007. DOI: $10.1029 / 2006 J F 000537$.

[9] M. Marani, A. D’Alpaos, S. Lanzoni, L. Carniello, and R. Rinaldo, "Biologically-controlled multiple equilibria of tidal landforms and the fate of the Venice lagoon," Geophys. Res. Lett., vol. 34, no. 11, p. L11402, Jun. 2007. DOI: 10.1029/2007GL030178.

[10] J. T. Morris, D. Porter, M. Neet, P. A. Noble, L. Schmidt, L. A. Lapine, and J. R. Jensen, "Integrating LIDAR elevation data, multi-spectral imagery and neural network modelling for marsh characterization," Int. J. Remote Sens., vol. 26, no. 23, pp. 5221-5234, Dec. 2005.

[11] C. Wang, M. Menenti, M. P. Stoll, E. Belluco, and M. Marani, "Mapping mixed vegetation communities in salt marshes using airborne spectral data," Remote Sens. Environ., vol. 107, no. 4, pp. 559-570, Apr. 2007.

[12] M. A. Lefsky, W. B. Cohen, G. G. Parker, and D. J. Harding, "LiDAR remote sensing for ecosystem studies," BioScience, vol. 52, no. 1, pp. 1930, Jan. 2002.

[13] P. H. Rosso, S. L. Ustin, and A. Hastings, "Use of LiDAR to study changes associated with Spartina invasion in San Francisco Bay marshes," Remote Sens. Environ., vol. 100, no. 3, pp. 295-306, Feb. 2006. 
[14] D. R. Streutker and N. F. Glenn, "LiDAR measurement of sagebrush steppe vegetation heights," Remote Sens. Environ., vol. 102, no. 1/2, pp. 135-145, May 2006.

[15] M. A. Brovelli, M. Cannata, and U. M. Longoni, "LiDAR data filtering and DTM interpolation within GRASS," Trans. GIS, vol. 8, no. 2, pp. $155-174$, Apr. 2004.

[16] A. Wehr and U. Lohr, "Airborne laser scanning—An introduction and overview," ISPRS J. Photogramm. Remote Sens., vol. 54, no. 2, pp. 68-82, Jul. 1999.

[17] K. Kraus and N. Pfeifer, "Determination of terrain models in wooded areas with aerial laser scanner data," ISPRS J. Photogramm. Remote Sens., vol. 53, no. 4, pp. 193-203, Apr. 1998.

[18] X. Yang, "Use of LiDAR elevation data to construct a high-resolution digital terrain model for an estuarine marsh area," Int. J. Remote Sens., vol. 26, no. 23, pp. 5163-5166, Dec. 2005.

[19] J. M. Montané and R. Torres, "Accuracy assessment of LiDAR saltmarsh topographic data using RTK GPS," Photogramm. Eng. Remote Sensing, vol. 72, no. 8, pp. 961-967, Aug. 2006.

[20] K. Zhang and D. Whitman, "Comparison of three algorithms for filtering airborne LiDAR data," Photogramm. Eng. Remote Sensing, vol. 71, no. 3, pp. 313-324, 2005.

[21] B. Petzold, P. Reiss, and W. Stossel, "Laser scanning-Surveying and mapping agencies are using a new technique for the derivation of digital terrain models," ISPRS J. Photogramm. Remote Sens., vol. 54, no. 2/3, pp. 95-104, Jul. 1999.

[22] J. Hyyppä, O. Kelle, M. Lehikoinen, and M. Inkinen, "A segmentationbased method to retrieve stem volume estimates from 3-D tree height models produced by laser scanners," IEEE Trans. Geosci. Remote Sens., vol. 39, no. 5, pp. 969-975, May 2001.

[23] M. Maltamo, K. Mustonen, J. Hyyppä, J. Pitkä, and X. Yu, "The accuracy of estimating individual tree variables with airborne laser scanning in a boreal nature reserve," Can. J. For. Res., vol. 34, no. 9, pp. 1791-1801, Sep. 2004.

[24] P. Ronnholm, J. Hyyppa, H. Hyyppa, H. Haggren, X. Yu, and H. Kaartinen, "Calibration of laser-derived tree height estimates by means of photogrammetric techniques," Scand. J. For. Res., vol. 19, no. 6, pp. 524-528, Dec. 2004.

[25] H. E. Andersen, R. J. McGaughey, and S. E. Reutebuch, "Estimating forest canopy fuel parameters using LiDAR data," Remote Sens. Environ., vol. 94, no. 4, pp. 441-449, Feb. 2005.

[26] M. E. Hodgson and P. Bresnahan, "Accuracy of airborne LiDAR-derived elevation: Empirical assessment and error budget," Photogramm. Eng. Remote Sensing, vol. 70, no. 3, pp. 331-339, 2004.

[27] A. Brzank and C. Heipke, "Supervised classification of water regions from LiDAR data in the Wadden Sea using a fuzzy logic concept," in Proc. ISPRS Workshop Laser Scanning SilviLaser, Espoo, Finland, Sep. 12-14, 2007

[28] D. Latypov, "Estimating relative LiDAR accuracy information from overlapping flight lines," ISPRS J. Photogramm. Remote Sens., vol. 56, no. 4, pp. 236-245, Jul. 2002.

[29] D. Shepard, "A two-dimensional function for irregularly spaced data," in Proc. 23rd ACM Nat. Conf., New York, 1968, pp. 517-524.

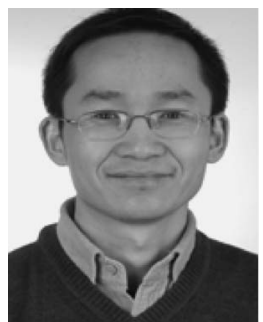

Cheng Wang received the Ph.D. degree in remote sensing from Université Louis Pasteur (ULP), Strasbourg, France, in 2005.

From November 2005 to April 2006, he was a Research Scientist with ULP. After that, he was as an Assistant Professor with the Chinese Academy of Sciences (CAS), Beijing, China, until April 2007. $\mathrm{He}$ is currently a Research Scientist with Boise Center Aerospace Laboratory, Idaho State University, Boise. His research interests include light detection and ranging and hyperspectral data processing and remote sensing for ecology applications.

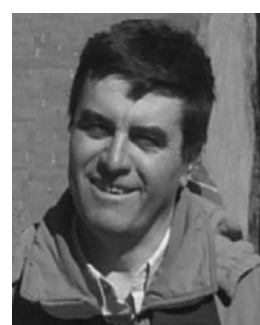

Massimo Menenti received the Laurea degree in physics from the Universitá di Roma, Rome, Italy, in 1972 and the Ph.D. degree in environmental sciences from the University of Wageningen, Wageningen, The Netherlands, in 1984

$\mathrm{He}$ was with Universitè Louis Pasteur, Strasbourg, France; with the Institute for Mediterranean Agriculture and Forest Systems, National Research Council, Italy, as a Director; with the Wageningen University and Reasearch Centre, Wageningen, The Netherlands; and with the Hydrospheric Sciences Laboratory, NASA Goddard Space Flight Center, Greenbelt, MD. He is currently a Full Professor of optical and laser remote sensing with Delft University of Technology, Delft, The Netherlands. His research interests include land surface hydrology and water management using Earth observation from space.

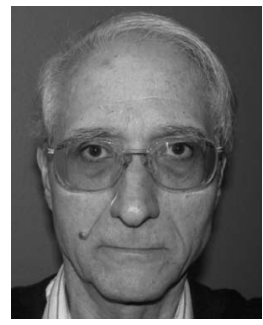

Marc-Philippe Stoll received the "Doctorat d'Etat" degree, with focus on magnetooptical properties, from the University of Strasbourg, Strasbourg, France, in 1973. He was first educated in physics and was involved in solid-state physics research.

From 1987 to 2004, he was the Head of the Radiometry and Remote Sensing Research Group, Laboratoire des Sciences de l'Image, de l'Informatique et de la Teledetection, Universite Louis Pasteur, Illkirch, France. In 1985, he joined the University of Strasbourg, Strasbourg, France, as a Full Professor. $\mathrm{He}$ is currently with the Universite Louis Pasteur. In the early 1980s, he turned to remote-sensing methods and Earth observations from satellites, with main research interests in thermal infrared, emissivity and surface temperature retrieval, and laser scattering by rough surfaces and granular media.

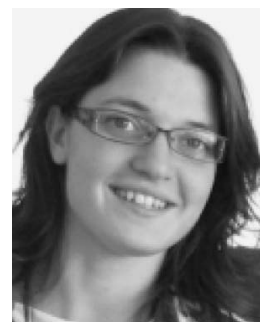

Alessandra Feola received the B.Sc. degree in environmental engineering and the Ph.D. degree in hydrodynamics and environmental modeling from the Università di Padova, Padova, Italy, in 2003 and 2007, respectively.

She is currently a Research Collaborator with the Superior Institute for Environmental Protection and Research, Venice, Italy. Her research interests include environmental monitoring and planning, ecological management, and restoration.

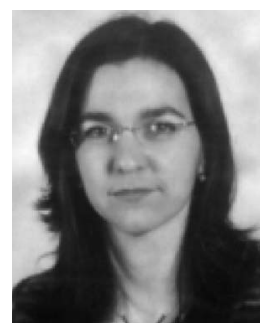

Enrica Belluco received the Laurea and Ph.D. degrees in environmental engineering from the Università di Padova, Padova, Italy, in 2001 and 2007.

She is currently a Research Collaborator with the Dipartimento di Inegegneria Idraulica Marittima Ambientale e Geotecnica, Università di Padova, Padova, Italy. Her research interests include remote sensing of tidal environments and ecogeomorphology analyses and modeling.

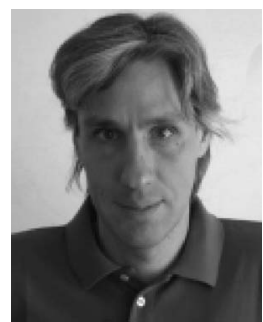

Marco Marani received the Laurea degree in civil engineering and the Ph.D. degree in hydrodynamics from the Università di Padova, Padova, Italy, in 1993 and 1997, respectively.

He was a Visiting Scientist with the Massachusetts Institute of Technology, Cambridge, between 1995 and 1996. He is currently an Associate Professor with the Dipartimento di Inegegneria Idraulica Marittima Ambientale e Geotecnica, Università di Padova, where he is the Director of the Research Center on Hydrology. His main research interests include biomorphodynamics of tidal environments; stochastic characterization and modeling of rainfall; and transport phenomena in hydrologic cycles. His research activity in these fields, in particular, also involves the development and application of remote-sensing observation techniques. 